\title{
Sextant
}

Revue de recherche interdisciplinaire sur le genre et la sexualité

36 | 2019

No children, no cry

\section{La matrice de la mémoire : non-maternité et traumatisme dans L'Espérance-macada de Gisèle Pineau}

Natacha D'Orlando

\section{CpenEdition}

Édition électronique

URL : https://journals.openedition.org/sextant/397

DOI : $10.4000 /$ sextant.397

ISSN : 2795-8736

Éditeur

Éditions de l'Université de Bruxelles

\section{Édition imprimée}

Date de publication : 1 décembre 2019

Pagination : 175-189

ISBN : 978-2-8004-1705-9

ISSN : 1370-267X

\section{Référence électronique}

Natacha D'Orlando, «La matrice de la mémoire : non-maternité et traumatisme dans L'Espérancemacada de Gisèle Pineau », Sextant [En ligne], 36 | 2019, mis en ligne le 01 novembre 2021, consulté le 08 décembre 2021. URL : http://journals.openedition.org/sextant/397 ; DOI : https://doi.org/10.4000/ sextant.397

\section{(ब) $\odot$}

La revue Sextant est mise à disposition selon les termes de la Licence Creative Commons Attribution Pas d'Utilisation Commerciale - Partage dans les Mêmes Conditions 4.0 International. 


\title{
La matrice de la mémoire : non-maternité et traumatisme dans \\ L’Espérance-macadam de Gisèle Pineau
}

\author{
Natacha D'OrLANDO
}

\begin{abstract}
Résumé :
Cet article propose une lecture de l'œuvre de la romancière guadeloupéenne Gisèle Pineau à partir du couple matrice/mémoire, et de son corolaire stérilitéltraumatisme. Nous verrons comment le rejet de la maternité y est pensé comme la trace latente d'une histoire non-résolue et comme la représentation métaphorique du souvenir traumatique. Cette mémoire ancrée au ventre se traduit d'abord par le retour du motif de l'infanticide esclave, représenté dans Femmes des Antilles : traces et voix et L'Espérance-macadam puis réactivé dans les récits du contemporain. Cette non-maternité violente porte un discours sur l'héritage mémoriel liant les femmes esclaves et contemporaines, d'une part, et un regard critique sur la violence des mondes post-abolition, d'autre part. Dans un deuxième temps, nous verrons comment la non-maternité est aussi représentée comme la réponse instinctive d'un corps mutilé, devenu infertile à la suite d'un événement traumatique, en particulier dans L'Espérance-macadam où l'infertilité d'Éliette signale le viol dont la protagoniste ne garde pourtant aucun souvenir. Dans les deux cas, la non-maternité met en crise la reproduction, qu'il s'agisse de la reproduction d'un passé, d'un système ou d'une lignée.
\end{abstract}

\begin{abstract}
:
This article explores the relationship between womb and memory, sterility and trauma, in the work of the Guadeloupean novelist Gisèle Pineau. Focusing on Femmes des Antilles : traces et voix and L'Espérance-macadam, we will see how the rejection of motherhood can be read as a metaphorical representation of a painful and traumatic history. Examining the use of infanticide in Pineau's contemporary narratives, we will first argue that the criminal slave mother is both the image of a painful past and a depiction of the great violence of post-slavery Guadeloupe. We will then see how Eliette's barrenness embodies the trauma of an untold family secret in L'Espérancemacadam. Eliette's sterility is together the consequence of the incestuous rape and its only recollection, since the protagonist herself forgot about the event. In both cases,
\end{abstract}


the rejection of motherhood puts reproduction into crisis, be it the reproduction of a past (through memory), of a system or of a lineage.

\section{Introduction}

Dans le domaine caribéen, comme ailleurs, maternité et féminité se codéfinissent, chacune étant conçue comme la condition de l'autre. Dans un article consacré aux figures féminines dans les contes et légendes antillais, Véronique Corinus rapporte ainsi un conte marie-galantais dont la morale témoigne de cette injonction à la maternité. Le jour du jugement dernier, quatre femmes se succèdent devant le Créateur pour faire le bilan de leur existence : une religieuse, une épouse fidèle, une jeune vierge et une mère célibataire de dix-sept enfants (tous de père différent). Si chacune des trois premières femmes a mené une vie vertueuse et morale, seule la dernière est envoyée au paradis par un Dieu qui la félicite d'avoir si bien fructifié de son vivant:

Sé’w ki fè byen, davwa Bondyé té dit: "Kwazè zòt, mété ti-moun à tè pou plen la tè», «C'est toi qui as respecté ma parole car j'avais bien affirmé : «croisez-vous (sic), multipliez-vous, remplissez la terre» $»^{1}$.

Qu'importe sa situation conjugale ou familiale, nous dit ce conte avec humour, la femme la plus vertueuse sera toujours celle qui a produit le plus d'enfants.

Parce qu'il est un conte et qu'il contient une forte charge ironique, ce récit mariegalantais condense à l'extrême les normes du devoir maternel, semblant indiquer à tort que la maternité célibataire n'est pas stigmatisée ou dévalorisée dans les sociétés caribéennes. Il est tout de même révélateur d'un rapport singulier à la maternité et à la construction de genre dans l'espace caribéen. La sociologue jamaïcaine Dorian Powell insiste sur cette singularité de la féminité caribéenne, construite davantage par rapport à la maternité qu'à la conjugalité : «Parenthood, even more than wifehood, is the important source of feminine identity $»^{2}$. Les constructions de genre, nous dit ici Dorian Powell, reposent davantage sur les structures familiales que sur les rapports conjugaux. C'est par l'enfant (biologique ou adoptif), et par la communauté qui reconnaît la femme comme mère, qu'est actée la conformité de genre, non par l'amant, le compagnon ou l'époux, dont la présence n'est d'ailleurs pas toujours assurée. La condition devient ensuite la norme, et la maternité se trouve érigée en destin universel féminin. La poétesse jamaïcaine Opal Palmer Adisa se souvient ainsi que, lors de ses jeux de petite-fille, elle ne pouvait s'imaginer adulte qu'en se représentant mère :

that $i$ would be a mother

was never a doubt

I imagined my brood

Assembled at the table

${ }^{1}$ V. Corinus, « Les femmes dans les contes et légendes antillais », in M.-R. LAFLeUR, Lang a fanm ou ce que le créole dit des femmes !, Matoury, Ibis Rouge, 2005, p. 96.

2 D. Powell, «Caribbean women and their response to familial experiences », Social and Economic Studies, 35/2, juin 1986, p. 122. 


\author{
Enjoying dinner \\ $\mathrm{Me}$ - the mother, directing \\ With tender care the \\ fabric of their lives \\ that we would be happy \\ that love was the foundation \\ the walls and even the ceiling. \\ That I would administer to \\ their every need was a given \\ motherhood was not a goal \\ rather the unwritten rule ${ }^{3}$.
}

La maternité est ici règle tacite mais partagée, norme sociale informulée qui structure l'imagination de la petite fille et informe ses représentations du futur. Par ailleurs, elle apparaît ici dans sa version idéalisée, la femme devenue mère semblant se fondre sans résistance dans le rôle assigné par son genre, sacrifiant avec bonheur ses aspirations personnelles pour construire un foyer aimant et harmonieux. Opal Palmer Adisa présente ici les deux biais par lesquels la maternité est érigée comme destin féminin : une pression sociale forte dans la socialisation des petites filles et une idéalisation de la maternité pour la leur rendre désirable.

\title{
Mère idéale et mère impossible
}

Cette survalorisation se traduit par deux paradigmes dans la mise en scène de la maternité : l'expérience maternelle peut être transformée en mythe ou caricaturée, dévalorisée, déconstruite dans ses valeurs. Dans le premier cas, c'est l'image d'une mère parfaite qui se perpétue, dans le second, c'est celle d'une femme en conflit avec la maternité, rejetant ses enfants ou l'éventualité de devenir mère. La première direction est majoritairement représentée par les littératures caribéennes masculines. Ces représentations se fondent sur une conception commune de la féminité et de la maternité, ou sur ce que Patrice DiQuinzio nomme la «maternité essentielle » ( « essential motherhood »), cette idéologie qui pense la mère et la femme comme deux concepts inséparables : douces, aimantes et attentives aux autres par nature, les femmes seraient irrémédiablement portées à s'accomplir dans la maternité 4 . Dans les littératures nourries de ces représentations, les deux seules places narratives libres pour les personnages féminins sont celles que les personnages masculins ne peuvent pas occuper, celles, donc, de l'amante ou de la mère. La valeur axiologique d'une protagoniste tient alors à sa capacité à s'investir dans sa fonction maternelle, à incarner les qualités associées à la maternité (tendresse, sens du sacrifice, dévouement) et à accompagner de loin les héros dans leurs quêtes et évolutions. La mère est alors le souvenir vaporeux des récits d'enfance, la mère-courage du second-plan, la pathétique et discrète présence accompagnant le héros sans trop lui faire d'ombre.

3 O. Palmer Adisa, « From Under the Tree to Running Home », in D. Smith Silva et S. A. James AleXander (eds.), Feminist and critical perspectives on Caribbean mothering, Trenton, Africa World Press, 2013, p. 40.

${ }^{4}$ P. DiQuinzio, The Impossibility of Motherhood: Feminism, Individualism and the Problem of Mothering, Hoboken, Taylor and Francis, 2013, p. xiii. 
Sans son enfant, rien ne justifie son existence. C'est en substance ce que proclame Délira, mère du héros Manuel dans le roman de Jacques Roumain Gouverneurs de la rosée. Lorsque meurt son fils, décrit dans le récit comme une figure messianique prête à sauver son village ravagé par le déboisement, la sécheresse, l'érosion des mornes et les conflits inter-familiaux, Délira semble perdre non pas le sens de son existence, mais jusqu'à la matière de son corps confondu dans celui de son fils :

Manuel, ah Manuel, tu étais mes deux yeux, tu étais mon souffle, tu étais mon sang : je voyais par tes yeux comme la nuit voit par les étoiles, je respirais par ta bouche, et mes veines se sont ouvertes quand ton sang a coulé, ta blessure m'a fait mal, ta mort m'a tuée. Je n'ai plus rien à faire sur la terre ${ }^{5}$.

Mère courage et sacrifiée, Délira n'existe que comme le miroir de la grandeur de son enfant. Ses besoins sont indexés à ceux de son fils, aussi, nous dit le roman, ne mange-t-elle que lorsque son fils et son époux ont été rassasiés, s'il reste à ce moment-là quelque chose pour remplir son assiette ${ }^{6}$. Une fois ce fils disparu, une fois mort celui qui donnait sens à sa présence, Délira n'a plus de fonction narrative, sinon à célébrer la grandeur du héros sacrifié ou à préserver son héritage en cachant qu'il a été assassiné, s'assurant ainsi que le village ne s'enflamme pas pour venger la mort du héros. Elle peut donc s'effacer au profit d'Anaïse, compagne de Manuel, dont on apprend dans les dernières lignes du roman qu'elle attend l'enfant du héros. Une mère en remplace ici une autre, et celle qui occupait la fonction de compagne trouve désormais à s'incarner dans la mère protectrice, vectrice et garante de la descendance héroïque de Manuel. La mère reste, dans les littératures caribéennes, la version la plus célébrée du féminin, une figure idéalisée et sacrificielle qui plane de loin sur de très nombreux récits ${ }^{7}$. Elle est aussi, selon l'écrivaine d'origine trinidadienne Dionne Brand, une présence littéraire envahissante qui assourdit toutes les autres apparitions du féminin et empêche l'émergence du « corps pour soi », d'un corps féminin qui ne soit ni maternel ni virginal, et que l'on représente pour autre chose que pour ce qu'il produit, symbolise, métaphorise ${ }^{8}$.

Ailleurs, pourtant, les représentations de la maternité comme destin féminin sont nettement plus ambivalentes. Un hiatus s'ouvre alors entre une norme genrée, que les protagonistes elles-mêmes formulent et intègrent, et la réalité de leurs désirs et expériences de la maternité Dans son essai sur les écrivaines antillaises de langue française, Maryse Condé remarque ainsi que le mythe maternel est rarement réutilisé par les écrivaines sans être remodelé ou critiqué. S'il existe bien « toute une littérature [qui] exalte l'enfantement, l'allaitement [et] magnifie l'attachement de la

5 J. Roumain, Gouverneurs de la rosée, Paris, Zuma, 2013 [1944], p. 184.

${ }^{6}$ Ibid., p. 98.

7 J. L. Liddell, « The Narrow Enclosure of Motherdom/Martyrdom: A Study of Gatha Randall Barton in Sylvia Wynter's The Hills of Hebron », in C. Boyce Davies et E. SAvory FIDo (eds.), Out of the Kumbla : Caribbean women and Literature, Trenton, N. J, Africa World Press, 1994, p. 321-330.

${ }^{8}$ D. Brand, «This body for itself », in D. Brand, Bread out of stone: Recollections on sex, recognitions, race, dreaming and politics, Toronto, Coach House Press, 1994, p. 47-49. 
mère à son petit ${ }^{9}$, nombre de romancières prennent aussi leurs distances avec cette littérature de célébration et choisissent de raconter des mères moins admirables et plus ambivalentes. Étudiant les œuvres de Simone Schwarz-Bart ou Michèle Lacrosil, Condé constate que les personnages féminins n'y parviennent pas à enfanter, refusent de devenir mères, ou se montrent distantes, mal-aimantes voire violentes avec leurs fil $\cdot$ le $\cdot s$, comme pour protester contre la faiblesse des perspectives qui leur sont offertes pour se réaliser. L'association entre féminin et maternel est ici utilisée à rebours, la déconstruction du modèle maternel servant à critiquer à plus large échelle les modèles et normes de genre imposés aux femmes.

L'écrivaine guadeloupéenne Gisèle Pineau met en scène ces maternités problématiques créant au fil de ses œuvres deux catégories de personnages maternels : les « mauvaises mères » et les femmes sans enfant. La première catégorie regroupe les mères imparfaites, abusives ou mal-aimantes, racontées le plus souvent du point de vue de l'enfant rejeté, qui tente de décrire une génitrice dont il peine à comprendre les réactions. Ce positionnement énonciatif est important parce que le lien d'affection, d'attentes, d'émotions qui relie l'enfant à la mère conditionne sa manière de la représenter. Qu'elle soit idéalisée ou caricaturée, la mère est toujours un portrait reconstitué par le fils ou la fille à partir de ses souvenirs les plus marquants et de ses propres sentiments d'abandon, de délaissement ou de désamour.

La « mauvaise mère » peut d'abord être la mère abandonneuse, comme l'est Pâquerette, génitrice de la narratrice Josette dans Fleur de Barbarie. Josette grandit loin d'une mère qui l'a abandonnée très jeune et ne rentre en contact avec elle que lors des quelques événements d'usage : anniversaires, Noël, ou d'autres rares occasions qui valent à sa fille un bref contact téléphonique ${ }^{10}$. L'abandon peut être aussi affectif, et non plus matériel, lorsque le personnage féminin se désinvestit émotionnellement de ses fil·le·s sans pour autant confier leur éducation à une personne extérieure. Dans Cent vies et des poussières, publié en 2012, Gina, la mère de la narratrice est par exemple habitée par un désir boulimique de maternité, une envie irrépressible de faire chaque année un nouvel enfant ${ }^{11}$. Lorsque le charme de la grossesse se rompt et que les nouveau-nés deviennent des individus autonomes, la fièvre de Gina retombe, elle n'est alors plus capable de les aimer suffisamment, considère ses enfants comme perdus et s'en désintéresse progressivement. À travers ces mères imparfaites, Gisèle Pineau interroge les sources du désir maternel, en particulier dans des situations où l'accès à la maternité est à la fois encouragé et réprouvé par les discours sur le genre, où les femmes doivent devenir mères pour être confirmées dans leur féminité mais s'exposent, lorsqu'elles le deviennent trop tôt, trop souvent ou en dehors du cadre du mariage, à de violentes réprimandes verbales ou physiques ${ }^{12}$. Dans le même temps,

${ }^{9}$ M. Condé, La Parole des femmes : essai sur des romancières des Antilles de langue française, Paris, L'Harmattan, 1979, p. 40.

${ }^{10}$ G. Pineau, Fleur de Barbarie, Paris, Mercure de France, 2005.

${ }^{11}$ G. Pineau, Cent vies et des poussières, Paris, Mercure de France, 2012.

${ }^{12}$ Sur cet apparent paradoxe entre injonction à la virginité et injonction à la maternité, voir en particulier O. SENIOR, Zigzag et autres nouvelles de la Jamaïque, trad. C. Raguet, Carouge-Genève, Éditions Zoé, 2010 [1995]. Cissy, la protagoniste de la nouvelle « Déchiffre les cœurs », subit longtemps les moqueries des gens de son village (et « même [de sa] propre 
Pineau réfléchit au devenir du modèle maternel dans des situations où les conditions du bien-être d'un enfant ne peuvent pas être assurées par la mère.

C'est sur la seconde catégorie de maternités problématiques que nous souhaitons aujourd'hui nous attarder, en évoquant les personnages de femmes infertiles, avortées ou infanticides, c'est-à-dire à la fois celles qui n'auront jamais d'enfant, celles qui n'en ont pas encore et celles qui n'en ont plus ${ }^{13}$. Dans les trois cas, les protagonistes gravitent en dehors du domaine de la féminité légitime, à des degrés plus ou moins importants selon qu'elles renoncent volontairement à avoir un enfant ou qu'elles soient physiologiquement incapables d'en porter, et selon que ce refus soit temporaire et circonstanciel ou qu'il soit définitif. Au sein de ces récits, la femme sans enfant est prise dans une série de conflits intérieurs et extérieurs : avec son corps, avec son genre, avec se(s) partenaire(s), avec sa mère, avec la lignée dans laquelle elle s'inscrit, avec son passé individuel ou avec l'histoire collective. C'est sur cette dernière forme de conflit que nous proposons aujourd'hui de nous arrêter, en interrogeant le lien entre non-maternité et mémoire dans l'œuvre de Gisèle Pineau. Nous proposons de lire ici le rejet de la maternité comme la réponse à une histoire collective, familiale ou individuelle non réglée et comme la représentation métaphorique d'un souvenir traumatique. Hantées par l'histoire de l'esclavage, par un secret familial ou par un drame individuel, les protagonistes travaillent à donner forme à leur futur en courtcircuitant les logiques de transmission d'un passé toxique. Infertilités volontaires et involontaires sont donc des réalités mémorielles, incarnations charnelles d'un passé irrésolu sur lequel ne peut se fonder nulle perspective d'avenir. Cette mémoire ancrée au ventre se traduit de deux manières, que je propose ici d'étudier successivement. L'association entre la non-maternité et l'histoire se signale d'abord par le retour d'un souvenir obsédant au fil des œuvres de Gisèle Pineau, celui de l'infanticide et de l'avortement des femmes esclaves, d'abord, et de leurs héritières contemporaines ensuite. L'esclave infanticide apparaît alors comme l'ancêtre incontournable et inquiétant, dont la présence continue de peser sur les imaginaires des mères et des fil $\cdot \mathrm{le} \cdot \mathrm{s}$ antillais $\cdot e \cdot s$ après plusieurs générations. À l'échelle individuelle, la non-maternité est aussi représentée comme la réponse instinctive d'un corps mutilé, devenu infertile à la suite d'un événement traumatique, comme nous le verrons dans L'Espérancemacadam. Dans les deux cas, la non-maternité met en crise la reproduction, qu'il s'agisse de la reproduction d'un passé, d'un système ou d'une lignée.

manman » (25) parce qu'elle est une des dernières de sa génération à ne pas avoir d'enfant. On la considère comme « bréhaigne » et donc inutile dans sa féminité. Lorsqu'elle tombe enceinte de son compagnon, sa mère lui reproche, à l'inverse, d'avoir fait comme toutes les jeunes femmes qui se laissent séduire et féconder par un « coq de basse-cour » (28), et d'avoir donné un enfant hors mariage à un homme déjà engagé ailleurs. Ces réprimandes ne l'empêchent pas de se réjouir de la grossesse de sa fille et de préparer joyeusement la venue du futur bébé. La mère participe par convention à la politique de respectabilité, mais elle se montre tolérante et compréhensive au moment où sa fille, comme tant d'autres, y désobéit.

${ }^{13}$ Il s'agit là des trois situations que les Grecs antiques désignaient par l'adjectif « apais ». $C f$. L. Bodiou, P. Brulé et L. Pierini, « En Grèce antique, la douloureuse obligation de la maternité », Clio. Femmes, Genre, Histoire, 21, 2005, p. 17-42. 


\section{Généalogies infanticides. Non-maternités esclaves dans Femmes des Antilles : traces et voix}

En 1998, Gisèle Pineau fait paraître avec l'historienne Marie Abraham un recueil intitulé Femmes des Antilles : traces et voix ${ }^{14}$. Dans ce travail composite se mêlent des récits historiques, des nouvelles semi-fictives et des témoignages de femmes contemporaines. Croisant les époques et les méthodes, Gisèle Pineau part sur les traces de femmes antillaises fictives ou réelles en se concentrant sur leur vécu individuel et leurs manières de composer avec les violences historiques, étatiques, conjugales dans lesquelles elles se trouvent prises. Une partie importante des récits de Femmes des Antilles est consacrée au souvenir de la Traite et de l'esclavage, depuis la capture des femmes en Afrique jusqu'à l'immédiat post-abolition, en passant par la traversée de l'Atlantique sur les bateaux négriers. Dans ce contexte d'esclavage, la maternité apparaît à la fois comme une fonction impossible, les mères ne pouvant ni protéger ni éduquer leurs enfants face à la violence esclavagiste, et comme une fonction indésirable parce qu'elle sert les intérêts du maître et du système plantationnaire. Là où l'enfant est une pièce de la propriété du maître, la maternité participe en effet d'une reproduction biologique, d'une part, et économique, d'autre part, la naissance d'un nouvel enfant assurant le renouvellement (sans frais) des forces de travail esclave et, plus largement, des systèmes coloniaux et esclavagistes ${ }^{15}$. La fécondité supposée d'une esclave conditionne alors sa valeur financière, comme l'évoque la narratrice de la nouvelle "Clarisse » se rappelant sa vente : "Ils mesuraient mes hanches et comptaient leurs sous en même temps que les négrillons qui sortiraient de mon ventre ${ }^{16}$. Transformée en une " machine à enfanter $»{ }^{17}$, Clarisse comprend qu'elle n'est que l'agente d'une reproduction dans laquelle elle-même ne reproduit rien : en tant qu'esclave, il ne lui est permis de rien transmettre aux enfants qu'elle fera naître, ni son nom, ni sa culture, ni la mémoire de l'Afrique d'où elle a été arrachée. C'est seulement le système esclavagiste qui doit se reproduire à travers elle, renouvelant une force de travail sans cesse menacée par le fort taux de mortalité parmi les femmes, hommes et enfants esclaves ${ }^{18}$.

Le refus d'enfanter prend dans cette situation une double valeur : il est à la fois une réponse individuelle à un événement traumatique et une tentative politique de saboter le système esclavagiste de l'intérieur. Que la grossesse soit évitée, écourtée ou que l'enfant soit sacrifié à la naissance, il est à la fois une manière d'agir contre l'esclavage et pour l'enfant (à qui est épargnée toute une vie de servitude), de résister

${ }^{14}$ M. Abraham et G. Pineau, Femmes des Antilles : traces et voix, Paris, Stock, 1998. Nous désignerons désormais le roman par ses initiales $F A$.

${ }_{15}$ M.-K. Fleming Miller, (Re)productions: Autobiography, Colonialism, and Infanticide, New York, Peter Lang, 2003.

${ }^{16}$ FA, p. 129.

${ }^{17}$ FA, p. 11.

18 Roberts considère, dans le cas des États-Unis, que moins de deux enfants esclaves sur trois atteignaient l'âge de dix ans. Cf. D. RoBerTs, Killing the Black Body, New York, Pantheon Books, 1997. 
aux intérêts du maître et d'abandonner face à la violence de son système ${ }^{19}$. Dans l'œuvre de Gisèle Pineau, infanticides et avortements esclaves sont toujours pensés dans une perspective transgénérationnelle, permettant de problématiser la transmission mémorielle à partir du parallèle implicite entre l'enfant mort ou non-né et le souvenir traumatique impossible à négocier. Dans la nouvelle «Zanina : la Traversée », la narratrice tente d'avorter pour se délester de la mémoire des viols subis pendant le voyage vers les Caraïbes :

J'ai enfoncé des bâtons dans mon corps

J'ai mangé de la terre et des herbes à poison.

Je me suis bourrée le ventre de coups de poing.

Et je me suis jetée contre terre.

L'enfant des hommes du bateau s'est jamais décroché ${ }^{20}$.

Quand la plupart des avortements évoqués par les littératures caribéennes reposent uniquement sur l'usage de plantes emménagogues, Zanina tente d'avorter par des méthodes invasives et particulièrement violentes. Pour ne pas laisser les « hommes du bateau » se reproduire à travers elle, elle rejoue la scène de son viol, frappe, pénètre et agresse son corps, retournant contre elle-même la violence subie pendant la Traversée ${ }^{21}$. L'avortement-agression signale l'intégration d'un souvenir traumatique, métaphorisé ici sous la forme du fœetus indésirable, qui envahit le corps et la mémoire de la narratrice incapable de décrocher la trace physique ou mémorielle de l'événement. Enfin, à un dernier niveau de signification, le récit de Zanina réactive la métaphore du viol fondateur dans les Caraïbes, figure de discours qui attribue l'origine des peuples antillais à la rencontre forcée entre un père européen et une mère africaine fécondée par contrainte ${ }^{22}$. Le corps de Zanina est aussi le corps de la mère

${ }^{19}$ Sur la résistance des femmes esclaves à la maternité, voir par exemple H. BECKLES, Natural rebels: A social history of enslaved black women in Barbados, New Brunswick, N. J., Rutgers University Press, 1989 ; A. Gauthier, Les Scurs de Solitude : femmes et esclavage aux Antilles du XVII au XIX ${ }^{e}$ siècle, Rennes, Presses universitaires de Rennes, 2010 ou, pour le contexte nord-américain, H. CARBY, Reconstructing womanhood: the emergence of the AfroAmerican Woman novelist, New York, Oxford, Oxford University Press, 1987.

${ }^{20} F A$, p. 91.

${ }^{21}$ En ce sens, le geste de Zanina rappelle d'autres récits dans lesquels un personnage violé reproduit son agression en s'en prenant à l'enfant qu'elle porte. Pensons particulièrement à Breath, Eyes, Memory, de l'écrivaine haïtienne Edwidge Danticat, où Martine plante un couteau dans son ventre au moment de sa seconde grossesse, incapable de s'affranchir du souvenir du viol qu'elle a subi plusieurs décennies plus tôt, pendant la dictature duvaliériste. E. DANTICAT, Breath, Eyes, Memory, New York, Vintage Books, 1994. Sur le lien entre souvenir traumatique et comportements auto-destructeurs, voir M. Braveheart-Jordan et L. DeBruyn, " So she may walk in balance : integrating the impact of historical trauma in the treatment of Native American Indian women », in J. AdLeman \& G. M. Enguidanos (eds.), Haworth innovations in feminist studies. Racism in the lives of women: Testimony, theory and guides to antiracist practice, New York, Harrington Park Press/Haworth Press, 1995, p. 345-368.

${ }^{22}$ S. Mulot, «Le mythe du viol fondateur aux Antilles françaises », Ethnologie française, 37/3, 2007, p. 521-524. Sur le sujet, voir aussi C. Rody, The daughter's return: Africainamerican and Caribbean women's fictions of History, New York, Oxford University Press, 2001, p. 112-114. 
violentée et fécondée par tous les hommes du bateau et qui tente en vain de courtcircuiter cette histoire en gestation.

Dans les récits contemporains, enfin, avortements et infanticides deviennent des souvenirs obsédants qui continuent à hanter les femmes et à informer leur rapport à la maternité. Au mythe du viol fondateur se substitue alors celui de l'infanticide originel, la première mère caribéenne apparaissant comme une mère impossible, forcée à rejeter une progéniture qu'elle ne peut ou ne veut assumer ${ }^{23}$. Ainsi, la mère esclave infanticide traverse les romans de Gisèle Pineau et sert d'outil de comparaison pour raconter les maternités contemporaines. Dans L'Espérance-macadam, le meurtre du nouveau-né de Glawdys apparaît comme une réactivation de l'infanticide originel :

Glawdys, la fille qui avait jeté son bébé au bas du pont des Nèfles n'y avait jamais cru, au bonheur. Elle avait fait comme ces Négresses des premiers voyages qui tuaient leurs nouveau-nés pour pas qu'ils naissent dans l'esclavage, tombent pas dans les pattes des négriers. La fille avait juste tiré son petit des griffes de Babylone, de ses mensonges, ses rêves dorés, son espérance-macadam et ses résurrections, ses actes sur papier timbré, ses lois, ses lumières, ses bourses, allocations, subventions...

La comparaison permet d'extraire le geste infanticide du cadre de la pathologie individuelle, du geste irrationnel et incompréhensible. Née d'un viol collectif, abandonnée par sa génitrice et élevée par une femme qui la garde enchaînée durant toute son enfance, Glawdys grandit dans une violence comparable à celle vécue par ses aïeules esclaves. Lorsqu'elle tue son nouveau-né, c'est moins par folie que dans un élan de froide lucidité sur le monde qui l'entoure, décidant de ne pas imposer sa violence à un nouveau-né innocent. Le retour du motif infanticide permet à Gisèle Pineau de nouer autour d'une généalogie commune la situation des femmes esclaves et celle des femmes contemporaines, de mettre en lumière la violence d'un monde qui continue à rendre la mort plus désirable que la survie. L'esclave infanticide devient alors un objet mémoriel, symbolisant le souvenir rejoué et réactivé d'une histoire féminine traumatique ${ }^{24}$.

\section{Stérilité, amnésie et traumatisme dans L'Espérance-macadam}

Paru en 1995, L'Espérance-macadam est le troisième roman Gisèle Pineau, après La grande drive des esprits publié en 1993 et Un papillon dans la cité, son premier roman jeunesse, édité en 1992. L'Espérance-macadam nous conduit sur les traces d'Éliette, habitante presque septuagénaire du quartier de Savane en Guadeloupe. Nous y suivons plus précisément sept jours de ses pensées et souvenirs, pris dans le mouvement de ce qu'Éliette nomme sa « maladie de la pensée ${ }^{25}$, une forme de

${ }^{23}$ Voir notamment A.-M. Sol, « Histoire(s) et traumatisme(s) : l'infanticide dans le roman féminin et antillais », The French Review, 81/5, avril 2008, p. 976-984. Pour les représentations littéraires de l'infanticide, voir M.-C. Agnant, Le Livre d'Emma, La Roque-d'Anthéron, Vents d'ailleurs, 2004 ; G. Nichols « Ala », in I is a long memoried woman, Londres, Karnak House, 1983 ou E. Trouillot, Rosalie l'Infâme, Paris, Dapper, 2003.

${ }^{24}$ Voir aussi G. Pineau, Morne Câpresse, Paris, Mercure de France, 2008.

${ }^{25}$ G. Pineau, L'Espérance-macadam, Paris, Stock, 1995, p. 21. Nous désignerons désormais le roman par ses initiales $E M$. 
rumination hypermnésique qui la conduit à rejouer obsessivement les souvenirs les plus tragiques et violents des soixante-et-une années passées depuis le cyclone de 1928. Éliette garde en mémoire tous les crimes de Savane : les épouses assassines, les amants féminicides, les viols collectifs et les infanticides. Toutes ces images traversent le récit, parfois par bribes, parfois intégralement, comme pris dans une narration cyclonique où chaque événement passe et repasse devant les yeux des lecteur/ice's. Au moment où s'ouvre le roman, Éliette s'apprête à découvrir le septième crime des damnés de Savane : les viols à répétition qu'inflige depuis plusieurs années le voisin Rosan à sa fille ainée Angela. Cette révélation liminale doit conduire Éliette sur le chemin de sa propre mémoire. Soixante ans plus tôt, lors du cyclone de 1928, Éliette a en effet été frappée par « La Bête », figure obscure et monstrueuse derrière laquelle se cache le géniteur d'Eliette qui, comme elle le découvre au fil du roman, la viola la nuit du cyclone. Je propose ici d'interroger la vacuité matricielle d'Éliette, cette infécondité inexpliquée, non comme une stérilité physiologique mais comme un refus non-formulé de devenir mère, comme un motif non pas biologique mais symbolique. C'est le corps qui parle en ne se laissant pas féconder, et Gisèle Pineau utilise la non-maternité comme l'image autour de laquelle se tissent les trames de la mémoire traumatique féminine.

L'Espérance-macadam est le récit du retour à la mémoire d'une narratrice caractérisée au début du roman par son extrême enclosure. Rien ne se laisse pénétrer entièrement chez Éliette, ni sa mémoire, ni sa maison, ni sa matrice irrémédiablement stérile malgré ses deux mariages. Comme elle le regrette dans ses dernières années, Éliette n'est en effet jamais parvenue à porter un enfant. Plusieurs explications sont avancées pour comprendre sa stérilité :

Je sais pas si c'est à cause de la poutre qui m'avait traversée le jour du Cyclone... Ou bien parce que j'avais pas connu d'homme avant mes trente-cinq ans. Quand j'ai rencontré Renélien, mon premier mari, c'était déjà trop tard ${ }^{26}$.

L'incapacité d'Éliette à concevoir un enfant peut d'abord être la conséquence de la blessure infligée à son ventre lors du passage du cyclone et dont elle ne guérit que grâce au travail d'Éthena, «gardienne d'un jardin de médecines ancestrales » qui travailla à « rem[ettre] [s] es chairs en place et dépose[r] l'oubli comme un onguent sur ses brûlures $\gg{ }^{27}$. Elle peut aussi être liée plus indirectement au passage de La Bête et à l'influence de cet événement sur son rapport aux hommes et à la sexualité. Pendant de longues années après son viol, Éliette évite le contact des hommes et déploie des stratégies pour se mettre à l'abri de ceux qui la courtisent. Dévorée par La Bête alors qu'elle n'était qu'une enfant, Éliette refuse désormais d'être consommée par des hommes qui cherchent « à cueillir tout ce qui germait en [elle] ${ }^{28}$ et elle préfère laisser sa « chair rassir ». La métaphore végétale porte ici dans deux directions : elle évoque d'abord l'association entre la fécondité humaine et la fécondité végétale, la femme étant l'arbre d'où naît le fruit/enfant ${ }^{29}$. Mais elle associe également la femme

\footnotetext{
26 EM, p. 217.

27 EM, p. 274.

${ }^{28} E M$, p. 25.

29 Sur cette association, voir aussi EM, p. 138.
} 
au fruit comestible qui, dans le cas d'Éliette, est consommé précocement, forcé à murir lorsque son père l'introduit malgré elle sur la scène de la sexualité ${ }^{30}$. Dans les deux cas, l'infertilité est directement liée au viol, soit parce qu'elle est la conséquence d'une mutilation physique, soit parce qu'elle découle d'un choix conscient pour sortir de la sexualité après y avoir été intégrée de force. En ce sens, l'infertilité apparaît aussi comme le choix informulé d'un corps qui tente de se "dé-genrer », de se détacher du statut de femme et de toutes les violences qu'implique ce statut. Éliette se considère « bel et bien créée inutilement ${ }^{31}$ parce qu'elle n'a jamais donné naissance, souscrivant à l'idée que « la femme stérile n'est pas ou plus à proprement parler une femme $»^{32}$ et qu' « on est femme quand on a enfanté ${ }^{33}$ seulement. Dans son cas, cette association femme/mère peut aussi être lue à rebours : c'est parce qu'elle ne souhaite pas être une femme qu'Éliette résiste dans son corps à la maternité. L'infertilité est la trace du conflit d'Éliette avec un genre qui l'exposa aux violences de son père et qui n'apparaît jamais que comme une malédiction pour les personnages féminins ${ }^{34}$.

Enfin, si Éliette n'a jamais porté d'enfant, c'est aussi parce que le souvenir traumatique de l'inceste l'envahit physiquement et émotionnellement :

Vécu soixante ans avec un cyclone niché en dedans d'elle comme un serpent qui étouffait tous les bébés qu'elle aurait pu porter, tous les poupons à qui elle aurait aimé donner ses tétés à sucer. Un cyclone qui avait terrassé l'amour en elle. Une bête longue comme un ver solitaire et sournois qui lui avait mangé les entrailles et la cervelle ${ }^{35}$.

«En dedans d'elle », Éliette abrite une série d'images évoquant son père violeur, figures monstrueuses et invasives qui la dévorent de l'intérieur. Envahie par le souvenir de La Bête, Éliette n'est capable de nourrir ni l'enfant qui viendrait occuper son ventre, ni l'amour maternel qu'il lui faudrait pour recueillir un·e fils·le d'adoption. Ce n'est en effet pas seulement le corps d'Éliette qui se refuse à la maternité : lorsque la jeune Glawdys est abandonnée par sa mère et que toutes les femmes de Savane, hypnotisées par la beauté du nourrisson, se disputent sa garde, Éliette n'ose s'avancer pour demander à accueillir la petite fille. Elle laisse alors « passer sa chance de

${ }^{30}$ Nous pouvons ici penser à l'expression « force-ripe », utilisées dans les Caraïbes anglophones pour désigner un $\cdot e$ jeune dont le comportement est considéré comme précoce et inadapté pour son âge, notamment parce qu'il serait trop sexualisé. $C f$. R. Allsopp, Dictionary of Caribbean English usage, Jamaica, University of the West Indies Press, 2009, p. 240.

${ }^{31} E M$, p. 62.

${ }^{32}$ F. Héritier, Masculin/Féminin : la pensée de la différence, Paris, Odile Jacob, 2012.

${ }^{33}$ F. Alibar et P. Lembeye-Boy, Le couteau seul... : la condition féminine aux Antilles, vol. II, Paris, Éditions caribéennes, 1983, p. 158. Voir aussi E. B. BRoDy, Sex, Contraception and Motherhood in Jamaica, Cambridge (Mass.), Harvard University Press, 1981, p. 164 : « If you are a barren woman all your days wasted » et V. FULLER, Unlike Normal Women, Londres, Women's Press, 1995.

${ }^{34}$ Autre exemple du rapport entre l'infertilité d'Éliette et le genre : Éliette et son deuxième époux Hector n'auront jamais d'enfant parce que Hector est incapable de consommer leur mariage. Face à Éliette, son sexe est comme « un pied-bois attaqué dans les racines par une vermine sans visage » $(E M$, p. 27). C'est aussi la masculinité d'Hector qui se trouve mise en échec dans cette relation.

${ }^{35} E M$, p. 280. 
mère $»^{36}$ et observe sans bouger Éloïse se saisir de Glawdys, puis l'enchaîner et la maltraiter durant toute son enfance. Un parallèle s'établit ici entre capacité mémorielle et capacité émotionnelle : Éliette garde sa maison et sa mémoire scellées pendant de longues années, et c'est seulement après avoir accepté d'accueillir Angela, sa jeune voisine violée par son père Rosan, après avoir entendu cette histoire qui rappelle tant la sienne, qu'elle peut renouer avec le souvenir enfoui et partir à la recherche des informations dissimulées par sa mère.

Enfin, Éliette s'extrait par sa non-maternité du champ de la reproduction biologique et symbolique. Dans L'Espérance-macadam, les mécanismes de reproduction, de transmission et d'héritage contiennent toujours une part d'ambivalence : c'est une malédiction, un souvenir traumatique, un legs de violence et de brutalité qui se transmet d'une génération à la suivante ${ }^{37}$. L'histoire de Rosan, Rosette et Angela met particulièrement en lumière l'ambivalence de la parenté, de la reproduction et de la lignée dans le contexte de violence qui caractérise Savane. Dans ce récit, les parents apparaissent comme des figures inquiétantes du fait de leur omnipotence et du mélange de haine et d'amour qu'ils nourrissent pour leurs enfants. Lorsque le père d'Angela la force à avoir des rapports sexuels avec lui, il le fait en incarnant pleinement sa fonction paternelle, non en s'en écartant :

Je suis ton papa, lui souffla-t-il, je suis ton papa! Chaque jour j'ai mis à manger dans cette case pour que tu tiennes debout. J'ai le droit d'aller sur ma monture avant les autres ! Je suis ton papa, Angela! Quand tu trouveras un homme pour te donner ça, tu ne penseras plus à ton papa. Alors, j'ouvre le chemin pour les autres ${ }^{38}$.

Parce qu'il a créé sa fille, argue Rosan, il peut la posséder physiquement et parce qu'il est en charge de son éducation, il doit la préparer à la venue de ses futurs amants. La paternité offre en ce sens un accès total au corps de l'enfant, transformé ici en un animal domestiqué, entièrement à la disposition de l'homme qui choisit de le «monter ${ }^{39}$. Enfin, le mécanisme de la transmission généalogique prend aussi ici une couleur inquiétante. Lorsque Rosan viole Angela, c'est aussi parce qu'il est l'héritier d'une lignée maudite d'hommes incestueux. Au fil du récit, Éliette comprend en effet que Rosan est le fils de La Bête et que le scénario qu'elle a donc vécu pendant l'enfance s'est reproduit entre Rosan et Angela ${ }^{40}$. Fille de La Bête et sœur de Rosan, Éliette porte

${ }^{36} E M$, p. 62.

${ }^{37}$ Voir aussi les réflexions sur la malédiction du peuple antillais, EM, p. 167 ou 241. La malédiction est un motif central de l'œuvre de Gisèle Pineau, en particulier dans Morne Câpresse, Cent vies et des poussières et Chair Piment, où chaque famille porte un secret qui, sans jamais s'énoncer ouvertement, se transmet de génération en génération et affleure dans la tare d'un'e de ses descendant $\cdot e \cdot s$.

${ }^{38} E M$, p. 223.

39 EM, p. 106-109.

${ }^{40}$ Cette malédiction se transmet aussi comme une prophétie auto-réalisatrice : enfant, Rosan a été rejeté par la grand-mère qui l'élevait parce qu'elle lui trouvait une trop grande ressemblance avec son père et considérait qu'il " portait une charge de malédiction » ( $E M$, p. 110). Rosan a donc grandi dans un environnement hostile et sans amour. Dans le corps d'Angela, il cherche à retrouver, dit-il, l'innocence et la douceur de cette enfance qu'il n'a pas connue et à s'y cacher " comme dans une cache où n'entraient pas les démons de son 
en son sang le germe d'une lignée monstrueuse, que son incapacité à procréer permet de ne pas prolonger. À la place de cette généalogie masculine court-circuitée, Éliette reconstitue sa propre lignée féminine en recueillant Angela. Reconnaissant sa jeune voisine comme la fille qu'une devineresse haïtienne lui avait jadis promise ${ }^{41}$, Éliette redéfinit son expérience de la maternité et reconnaît que les liens mère/fille ne se constituent pas uniquement par le ventre. Ce faisant, elle remplace aussi la généalogie des bourreaux (où le père violeur donne naissance à un fils qui le sera à son tour) par une généalogie des victimes, rassemblées autour d'une expérience commune de la violence et un même cheminement vers la survie. L'infertilité d'Éliette dit donc à la fois le conflit se jouant entre la narratrice et son passé, et la manière dont elle travaille à s'extraire de la généalogie maudite dans laquelle elle fut intégrée par son viol.

Nous le voyons, le refus (conscient ou inconscient) d'enfanter lance un défi à la reproduction et à l'articulation entre passé et présent dans ces récits. Les femmes sans enfant, avortées ou infanticides métaphorisent une transmission mémorielle complexe, non-linéaire, rendue impossible par les histoires collectives et individuelles de chaque protagoniste. La non-maternité n'est donc pas chez Pineau un phénomène caractéristique du contemporain, comme elle peut l'être chez les théoriciens du childless, à l'image d'Ulrich Beck qui considère le phénomène comme symptomatique des valeurs de notre société actuelle ${ }^{42}$, ou Anne Gotman qui affirme que « l'individu volontairement sans enfant est résolument moderne en ce qu'il conçoit la vie comme un projet, comme un devenir, jamais comme un héritage à transformer ${ }^{43}$. Elle est plutôt un symptôme de l'histoire caribéenne, un trait caractéristique de cet espace au fil des époques et un motif significatif pour penser l'héritage mémoriel féminin.

\section{Bibliographie}

Abraham M. et Pineau G., Femmes des Antilles : traces et voix, Paris, Stock, 1998. Agnant M.-C., Le Livre d'Emma, La Roque-d'Anthéron, Vents d'ailleurs, 2004.

Alibar F. et Lembeye-Boy P., Le couteau seul... : la condition féminine aux Antilles, Paris, Éditions caribéennes, 1983.

Allsopp R., Dictionary of Caribbean English usage, Jamaica, University of the West Indies Press, 2009.

BЕск U., La société du risque. Sur la voie d'une autre modernité, Paris, Flammarion, 2008 .

Beckles H., Natural rebels: A social history of enslaved black women in Barbados, New Brunswick, N. J., Rutgers University Press, 1989.

enfance ». C'est donc pour échapper à ce souvenir de la violence que Rosan fait subir à Angela une violence encore plus destructrice. $C f . E M$, p. 253.

${ }^{41}$ Ibid., p. 11.

${ }^{42}$ U. BECK, La société du risque. Sur la voie d'une autre modernité, Paris, Flammarion, 2008, p. 257.

${ }^{43}$ A. Gotman, Pas d'enfant. La volonté de ne pas engendrer, Paris, Éditions de la Maison des sciences de l'homme, 2017, p. 228. 
Bodiou L., Brulé P. et Pierini, L., « En Grèce antique, la douloureuse obligation de la maternité », Clio. Femmes, Genre, Histoire, 21, 2005, p. 17-42.

BRAND D., "This body for itself ", in D. BRAnD, Bread out of stone: Recollections on sex, recognitions, race, dreaming and politics, Toronto, Coach House Press, 1994, p. 47-49.

Braveheart-Jordan M. et DeBruyn L., « So she may walk in balance: integrating the impact of historical trauma in the treatment of Native American Indian women ", in Adleman J. et Enguidanos G. M. (eds.), Haworth innovations in feminist studies. Racism in the lives of women: Testimony, theory and guides to antiracist practice, New York, Harrington Park Press/Haworth Press, 1995.

Brody E.-B., Sex, Contraception and Motherhood in Jamaica, Cambridge (Mass.), Harvard University Press, 1981.

CARBY H., Reconstructing womanhood: the emergence of the Afro-American Woman novelist, New York, Oxford, Oxford University Press, 1987.

Condé M., La Parole des femmes : essai sur des romancières des Antilles de langue française, Paris, L'Harmattan, 1979.

Corinus V., « Les femmes dans les contes et légendes antillais », in M.-R. LAFLEUR, Lang a fanm ou ce que le créole dit des femmes !, Matoury, Ibis Rouge, 2005.

Danticat E., Breath, Eyes, Memory, New York, Vintage Books, 1994.

DiQuinzio P., The Impossibility of Motherhood: Feminism, Individualism and the Problem of Mothering, Hoboken, Taylor and Francis, 2013.

Fleming Miller M.-K., (Re)productions: Autobiography, Colonialism, and Infanticide, New York, P. Lang, 2003.

Fuller V., Unlike Normal Women, Londres, Women's Press, 1995.

Gauthier A., Les Scurs de Solitude : Femmes et esclavage aux Antilles du XVII au XIX ${ }^{e}$ siècle, Rennes, Presses universitaires de Rennes, 2010.

Gotman A., Pas d'enfant. La volonté de ne pas engendrer, Paris, Éditions de la Maison des sciences de l'homme, 2017.

Héritier F., Masculin/Féminin : la pensée de la différence, Paris, Odile Jacob, 2012.

Liddell J. L., « The Narrow Enclosure of Motherdom/Martyrdom: A Study of Gatha

Randall Barton in Sylvia Wynter's The Hills of Hebron », in C. Boyce DavieS et E. Savory Fido (eds.), Out of the Kumbla: Caribbean women and Literature, Trenton, N. J, Africa World Press, 1994.

Mulot S., " Le mythe du viol fondateur aux Antilles françaises », Ethnologie française, 37/3, 2007, p. 521-524.

Nichols G., I is a long memoried woman, Londres, Karnak House, 1983.

Palmer Adisa O., «From Under the Tree to Running Home », in D. Smith Silva et S. A. James Alexander (eds.), Feminist and critical perspectives on Caribbean mothering, Trenton, Africa World Press, 2013.

Pineau G., L'Espérance-macadam, Paris, Stock, 1995.

Pineau G., Fleur de Barbarie, Paris, Mercure de France, 2005.

Pineau G., Morne Câpresse, Paris, Mercure de France, 2008.

Pineau G., Cent vies et des poussières, Paris, Mercure de France, 2012.

Powell D., « Caribbean women and their response to familial experiences », Social and Economic Studies, 35/2, juin 1986. 
Roberts D., Killing the Black Body, New York, Pantheon Books, 1997.

Rody C., The daughter's return: Africain-american and Caribbean women's fictions of History, New York, Oxford University Press, 2001, p. 112-114.

Roumain J., Gouverneurs de la rosée, Paris, Zuma, 2013 [1944].

Senior O., Zigzag et autres nouvelles de la Jamaïque, trad. C. Raguet, CarougeGenève, Éditions Zoé, 2010 [1995].

Sol A.-M., " Histoire(s) et traumatisme(s) : l'infanticide dans le roman féminin et antillais », The French Review, 81/5, avril 2008, p. 976-984.

Trouillot E., Rosalie l'Infâme, Paris, Dapper, 2003. 